\title{
The EXPANd trial: effects of exercise and exploring neuroplastic changes in people with Parkinson's disease: a study protocol for a double-blinded randomized controlled trial
}

Erika Franzén ${ }^{1,2,3^{*}}$, Hanna Johansson 1,2, Malin Freidle ${ }^{1}$, Urban Ekman ${ }^{3,4,5}$, Martin Benka Wallén ${ }^{1}$, Ellika Schalling ${ }^{6,7}$, Alexander Lebedev ${ }^{8,9}$, Martin Lövdén ${ }^{8}$, Staffan Holmin ${ }^{9}$, Per Svenningsson ${ }^{9}$ and Maria Hagströmer ${ }^{1,2,10}$

\begin{abstract}
Background: Parkinson's disease (PD) affects many physiological systems essential for balance control. Recent studies suggest that intensive and cognitively demanding physical exercise programs are capable of inducing plastic brain changes in PD. We have developed a highly challenging balance training (the HiBalance) program that emphasizes critical aspects of balance control through progressively introducing more challenging exercises which incorporates dual-tasking. Earlier studies have shown it to be effective in improving balance, gait and dual-tasking. The study design has thereafter been adjusted to link intervention-induced behavioral changes to brain morphology and function. Specifically, in this randomized controlled trial, we will determine the effects of the HiBalance program on balance, gait and cognition and relate this to task-evoked functional MRI (fMRI), as well as brain-derived neurotrophic factor (BDNF) in participants with mild-moderate PD.

Methods: One hundred participants with idiopathic PD, Hoehn \& Yahr stage 2 or 3, $\geq 60$ years of age, $\geq 21$ on Montreal Cognitive Assessment will be recruited in successive waves and randomized into either the HiBalance program or to an active control group (the HiCommunication program, targeting speech and communication). Both interventions will be performed in small groups, twice a week with $1 \mathrm{~h}$ sessions for 10 weeks. In addition, a 1 $h$, once a week, home exercise program will also be performed. A double-blinded design will be used. At the preand post-assessments, participants will be assessed on balance (main outcome), gait, cognitive functions, physical activity, voice/speech function, BDNF in serum and fMRI (3 T Philips) during performance of motor-cognitive tasks.

Discussion: Since there is currently no cure for PD, findings of neuroplastic brain changes in response to exercise would revolutionize the way we treat PD, and, in turn, provide new hope to patients for a life with better health, greater independence and improved quality of life.
\end{abstract}

Trial registration: ClincalTrials.gov: NCT03213873, first posted July 11, 2017.

Keywords: Balance, Cognition, Dual-task, Exercise, Gait, Imaging, Magnetic resonance neural growth factor, Neuroplasticity, Parkinson's disease

\footnotetext{
* Correspondence: erika.franzen@ki.se

'Department of Neurobiology, Care sciences and Society, Division of

Physiotherapy, Karolinska Institutet, Stockholm, Sweden

${ }^{2}$ Karolinska University Hospital, Allied Health Professionals Function, Function

Area Occupational Therapy \& Physiotherapy, Stockholm, Sweden

Full list of author information is available at the end of the article
}

(c) The Author(s). 2019 Open Access This article is distributed under the terms of the Creative Commons Attribution 4.0 International License (http://creativecommons.org/licenses/by/4.0/), which permits unrestricted use, distribution, and

reproduction in any medium, provided you give appropriate credit to the original author(s) and the source, provide a link to the Creative Commons license, and indicate if changes were made. The Creative Commons Public Domain Dedication waiver (http://creativecommons.org/publicdomain/zero/1.0/) applies to the data made available in this article, unless otherwise stated. 


\section{Background}

Parkinson's disease (PD) is a neurodegenerative disorder with no curative treatment and devastating impact on quality of life of patients and their caregivers, as well as a burden to the health care system [1-3]. It affects the substantia nigra and striatum in the basal ganglia,

which are among the most important structures in the central nervous system playing crucial roles in motor control, learning and high-order cognitive functions [4-6]. In addition, since the basal ganglia have many connections to the rest of the brain, several regions are secondarily affected by the disease. Gait and balance difficulties are prominent early in the disease and are associated with significant disability, decreased quality of life and falls [7]. Cognition, especially executive functions, is often altered in people with PD and characterized by deficits in attention, set shifting, planning, inhibitory control and dual-task (DT) performance [4]. There is also an important connection between gait / balance and cognitive function in people with PD [8] when investigated through the DT paradigm i.e., the performance of a motor task, often gait, concomitant with a cognitive task [9].

Levodopa, considered the gold-standard treatment for PD, has variable effects on cognitive function $[4,10]$. Likewise, gait and balance symptoms are just partially alleviated, or even non-responsive, with dopaminergic medication [11-13]. This highlights the importance of non-medical interventions in people with PD. Several systematic reviews and meta-analyses have found various types of exercise to improve a wide range of motor symptoms in PD, including gait ability, balance and strength [14-16]. In addition, promising effects of physical exercise on cognition have been demonstrated in people with PD [17-19]. However, the behavioral effects of exercise on both gait and cognition, as well as dualtask outcomes need further exploration. Above this, it is important to study the underlying neural structures and networks of the brain, especially establishing if, and how, it is altered by exercise [20].

A growing body of research highlights the role of exercise as an essential part of managing PD through potential neuroprotective mechanisms [21]. Multiple structural and physiological mechanisms have been suggested to underlie neuroplastic changes due to exercise in PD, such as increased synaptic strength and a preservation of dopamine neurons [21, 22]. Furthermore, exercise can induce general brain health that might also influence structural and functional properties of the brain [21]. As of yet, studies exploring exercise-induced brain changes in people with PD have been small-scaled and uncontrolled, and have rarely led from pilot studies to full scale randomized controlled trials (RCT). Further, there is missing or underreporting of correlation analysis between improvements in the behaviour and structural / functional improvements in the brain after an exercise intervention, prohibiting firm conclusions to be made.

We previously developed a group training program (the HiBalance program) that combines challenging balance exercises with additional cognitive and motor tasks (dual-tasking) in a progressive manner [23]. The results of a RCT conducted in a hospital research setting showed that participants in the HiBalance program significantly improved their balance and gait, as well as improved cognitive processing during walking compared to a passive control group $[19,24]$. Clinical effectiveness was further confirmed after adapting the program from three to two therapist-led sessions weekly and implemented across various clinical settings (in manuscript). As a final evaluative step, we wish to explore whether improvements in gait, balance and cognitive processing could be linked to neuroplastic changes in a doubleblinded design with an active control group. The feasibility of the proposed RCT design has been tested and adapted through a pilot study (in manuscript).

Hence, the purpose of this study is to determine the effects of a highly challenging balance training (the HiBalance program) on balance performance (primary outcome), gait ability and cognitive function (secondary outcomes) in comparison to an active control group receiving a speech and communication intervention in people with mild-moderate PD. Thereafter, we aim to relate the effects seen in behavior (balance, gait and cognition) to changes in functional connectivity, as well as blood markers of neurobiological plasticity. Our underlying hypotheses is 1) that the highly challenging exercise will lead to improved balance performance, gait ability $[16,24]$ and cognitive function $[18,19]$ and 2$)$ that the improvements seen in the behavior (balance, gait and cognition) after exercise will be related to altered activity in brain regions that rely on dopaminergic neurotransmission such as the fronto-striatal circuits involved in motor- cognitive control. Finally, we also expect that increased levels of brain-derived neurotrophic factor (BDNF) in serum after the intervention will be related to behavioral changes.

\section{Methods \\ Trial design}

The EXPANd (EXercise in PArkinson's disease and Neuroplasticity) Trial is a randomized controlled trial with a double-blinded design (registered at clinicaltrials.gov NCT03213873). The intervention group will receive the HiBalance program, while the control group receive a speech and communication treatment (HiCommunication) with a similar dose, as well as study setting and deliverance. Reporting of the study follows the SPIRIT 
(Standard Protocol Items: Recommendations for Interventional Trials) 2013 statement and guidelines.

\section{Study setting}

The assessments will be performed in a university setting with the interventions delivered in a university (academic) hospital setting in Stockholm, Sweden.

\section{Recruitment and screening}

Participants will be recruited through several sources, such as Karolinska University Hospital and other clinics in the vicinity, as well as via announcements in relevant forums, such as the Swedish Parkinson Association and newspapers. Recruitment will be performed in four successive waves, and participants will be randomly assigned (1:1 allocation ratio) to the intervention or the control group in blocks of approximately 25 participants per wave. In each wave, there will be two parallel groups of the intervention, as well as two parallel groups of the active control group. Each group will consist of 6 to 8 participants. Potential participants will initially be screened via telephone to determine eligibility before being asked to visit Karolinska Institutet for a full eligibility exam, see Fig. 1.

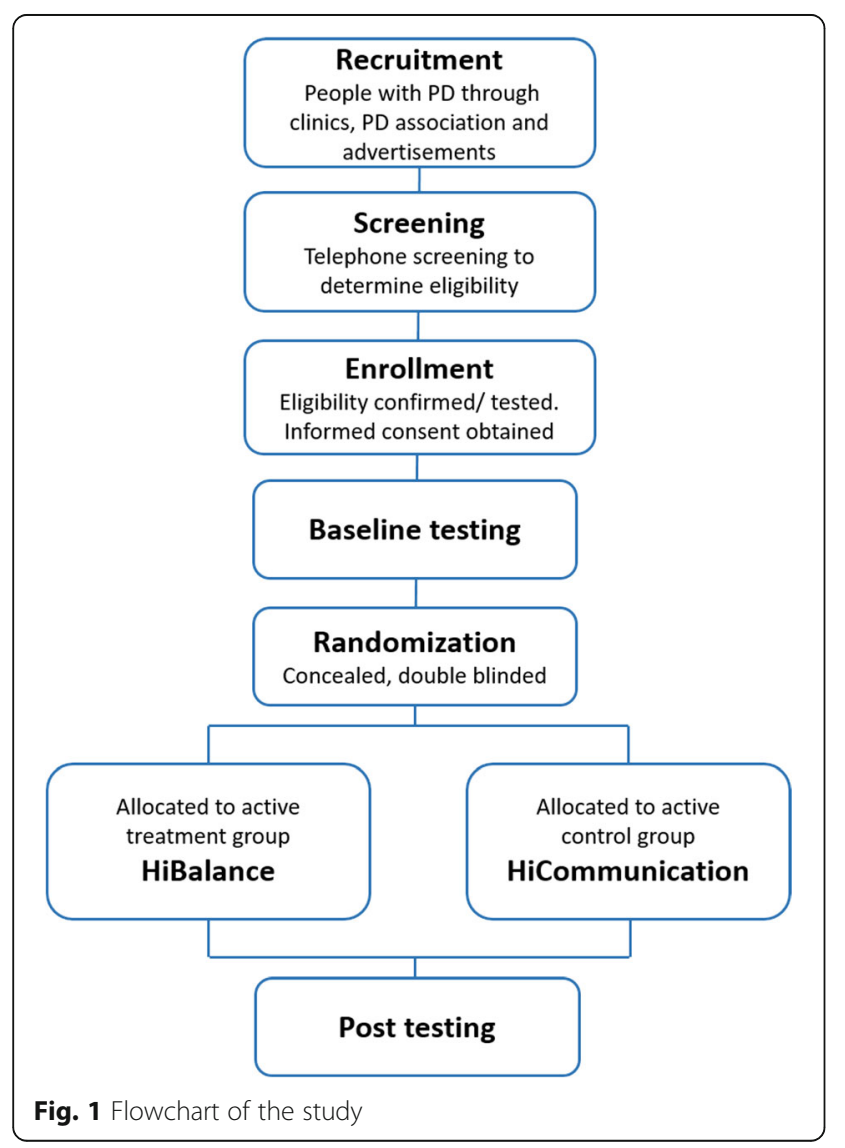

\section{Eligibility criteria}

Inclusion and exclusion criteria are compiled in Table 1.

\section{Assignment of intervention}

Allocation to the intervention or the control group will be blinded to the assessors at all time points, and participants will also be blinded to their group (treatment or control) allocation i.e., the two interventions will be presented as of equal interest in the study. The randomized sequence will be generated on randomization.com by a researcher independent to the project, and participants will receive an opaque envelope with the assigned group following the pre-testing sessions. All information on participants' group allocation and treating trainers will be kept confidential and non-disclosed from the assessors. At completion of each post-assessment, the assessors will answer a questionnaire to investigate the success of the concealment of the group allocation [27].

\section{Interventions \\ Intervention group - the HiBalance program}

The HiBalance program is based on scientifically wellestablished principles of exercise training and postural control, as well as current research on exercise in people with PD [23, 24, 28-31]. In brief, the HiBalance program targets four main components of balance control affected in PD (stability limits, anticipatory postural adjustments, sensory integration and motor agility) by principles of motor learning, i.e. specificity, progressive overload and variation, see Table 2 . The program also incorporates DT-exercises that gradually integrate cognitive tasks (e.g. counting or remembering items) and motor tasks (e.g. carrying or manipulating an object). To ensure highly challenging exercises, each task is individually adjusted, e.g. by altering the base of support, increasing movement speed/amplitude, restricting vision and varying the grade of multitasking. The difficulty level will be increased in three consecutive blocks, and intermittent presence of reactive postural adjustments will be used as an indicator of the appropriate difficulty level of exercises. Transfer effects and generalization of motor skills to everyday activities will be facilitated by training variation, integrating exercises from the main components with DT exercises. The training will be performed in a clinical setting for $1 \mathrm{~h}$, twice a week for a total of 10 weeks, as a group intervention ( 6 to 8 participants), and facilitated by two trained physical therapists. In addition, a home exercise program, focusing on aerobic capacity, lower extremity strength and core muscles, will be performed unsupervised in the participants home environment for $1 \mathrm{~h}$ once a week [29, 30]. Adherence to the intervention is registered by the trainers. 
Table 1 Study exclusion and inclusion criteria

\begin{tabular}{ll}
\hline Inclusion criteria & Exclusion criteria \\
\hline - Idiopathic PD & - Any other existing disorder that may substantially influence balance performance, voice or speech \\
- Hoehn \& Yahr [25] scores 2 to 3 & performance or participation in the interventions \\
- Stable dose of anti-Parkinson's medication for & - Having participated in an intensive exercise program for balance or speech during the last six \\
approximately 3 weeks & months \\
- $\geq 60$ years of age & For imaging \\
- Montreal Cognitive Assessment (MoCA) [26] & - Pacemakers, deep brain stimulators or other MRI incompatible implants \\
score $\geq 21$ & - Claustrophobia \\
- Ambulate indoors without mobility aid & - Unilateral or bilateral blindness \\
- Balance impairments ( $\leq 27$ on the Mini- & - Inability to hear instructions without hearing aid \\
BESTest) & - Severe states of: diplopia, tremor, dyskinesia or dystonia \\
\hline
\end{tabular}

\section{Control group - the HiCommunication program}

The control group will also receive a group treatment, the HiCommunication program, recently developed at Karolinska Institutet/Karolinska University Hospital. The program targets voice and speech changes associated with $\mathrm{PD}$, specifically aimed at voice intensity, voice quality and articulatory precision [32]. HiCommunication has the same dose as the HiBalance program $(1 \mathrm{~h}$, twice a week for 10 weeks) with a $1 \mathrm{~h}$ once weekly home exercise program. The program is performed by a speech-language pathologist in groups of 6 to 8 participants. The treatment will aim at increasing vocal loudness and improving articulatory precision, targeting four core areas (voice intensity, articulation, word retrieval and memory). Level of difficulty will be gradually increased in three blocks by progressing from using loud voice and clear speech in short and automatized utterances to using the same technique in more complex sentences and situations, see Table 2. The purpose of gradually increasing the cognitive load while performing the speech training is to enhance transfer of improved speech function to contexts beyond the clinical setting [33]. This intervention will be performed in a sitting position as opposed to the HiBalance intervention that is performed in standing and walking. The home exercise program, to be performed in the home environment for $1 \mathrm{~h}$ per week, will focus on relaxation and breathing exercises, as well as voice and speech exercises with additional word retrieval- and memory tasks.

\section{Adverse events}

Adverse events during the intervention period will be reported by the trainers to the research group, using a written form used in other intervention studies [24, 30]. Injurious falls and harmful adverse event will also be reported according to the routines at Karolinska University Hospital.

\section{Outcomes and procedure}

All assessments will be conducted during the ON phase of levodopa medication and at the same time of day at pre- and post-intervention assessments to restrict the influence of medication fluctuations. The assessments will be performed on three different sessions spread across three separate days to minimize fatigue. A general cognitive assessment (MoCA) and assessment of disease

Table $\mathbf{2}$ Overview of the main components and the progression of the interventions

\begin{tabular}{|c|c|c|c|c|}
\hline \multirow[t]{2}{*}{ Intervention } & \multirow{2}{*}{$\begin{array}{l}\text { Main } \\
\text { Components }\end{array}$} & \multicolumn{3}{|l|}{ Progression (blocks) } \\
\hline & & $\begin{array}{l}\text { A } \\
\text { week 1-2 }\end{array}$ & $\begin{array}{l}\text { B } \\
\text { week 3-6 }\end{array}$ & $\begin{array}{l}\text { C } \\
\text { week 7-10 }\end{array}$ \\
\hline $\begin{array}{l}\text { HiBalance } \\
\text { (intervention } \\
\text { group) }\end{array}$ & $\begin{array}{l}\triangleright \text { Sensory } \\
\text { integration } \\
\triangleright \\
\text { Anticipatory } \\
\text { postural } \\
\text { adjustments } \\
\triangleright \text { Motor } \\
\text { agility } \\
\triangleright \text { Stability } \\
\text { limits }\end{array}$ & $\begin{array}{l}\text { Exercises with focus on movement } \\
\text { quality, familiarization of the exercises } \\
\text { and task-specific motor learning. Sin- } \\
\text { gle task performance of exercises } \\
\text { pertaining to each of the main } \\
\text { components. }\end{array}$ & $\begin{array}{l}\text { Increased level of difficulty of the } \\
\text { exercises. Variation of the exercises } \\
\text { within the components. } \\
\text { Introducing cognitive and motor } \\
\text { dual tasks to increase the } \\
\text { complexity of the exercises. }\end{array}$ & $\begin{array}{l}\text { Complexity increased by task } \\
\text { variation and combining exercises } \\
\text { from all four main components, and } \\
\text { by integrating simultaneous } \\
\text { cognitive and motor dual tasks. }\end{array}$ \\
\hline $\begin{array}{l}\text { HiCommunication } \\
\text { (control group) }\end{array}$ & $\begin{array}{l}\triangleright \text { Voice } \\
\text { intensity } \\
\triangleright \\
\text { Articulatory } \\
\text { precision } \\
\triangleright \text { Word } \\
\text { retrieval } \\
\triangleright \text { Memory }\end{array}$ & $\begin{array}{l}\text { Exercises with focus on breathing, } \\
\text { phonation and articulation. } \\
\text { Establishing increased vocal loudness } \\
\text { while maintaining good voice quality }\end{array}$ & $\begin{array}{l}\text { Increased level of difficulty of the } \\
\text { exercises. Introducing memory } \\
\text { games and associational tasks to } \\
\text { increase cognitive load during } \\
\text { exercises. }\end{array}$ & $\begin{array}{l}\text { Complexity increased by increasing } \\
\text { difficulty of memory games, } \\
\text { incorporating more interaction } \\
\text { between participants and by adding } \\
\text { background noise. }\end{array}$ \\
\hline
\end{tabular}


severity (Hoehn \& Yahr) will be performed to confirm eligibility. Levodopa equivalent daily dose (LED) will be recorded and calculated according to Tomlinson et al. [34]. In addition, height, weight, sex and age, as well as questions regarding prior falls, walking aids and other diseases/disorders will be collected.

First, assessment of gait and balance performance, as well as questionnaires on walking ability, balance confidence, physical activity, anxiety and depression and health related quality of life will be performed. The order of the physical assessments (balance and gait) will be randomised for each participant at both pre- and posttesting to avoid systematic bias due to fatigue. Thereafter, one session will be dedicated to the evaluation of cognitive performance and speech and voice function, and the other to brain imaging. Each session will take 1.5-2 $\mathrm{h}$. In addition, blood will be sampled and habitual physical activity will be assessed at one week before and after the intervention in participants' home environment using an activity monitor.

\section{Primary outcome}

The primary outcome of the effect of the HiBalance program will be balance performance assessed with the Mini Balance Evaluation Systems Test (Mini-BESTest), which is a 14 item scale (maximal score 28) assessing four subsystems of balance control (anticipatory postural adjustments, reactive postural control, sensory orientation and dynamic gait) [35, 36]. This scale has been validated for people with PD [37-39].

\section{Secondary outcomes}

Gait will be assessed under single and DT conditions using an electronic walkway system (GAITRite, active zone: $8.3 \mathrm{~m}$, CIR Systems, Inc., Havertown, PA, USA), measuring temporal and spatial gait parameters. During DT gait, the Auditory Stroop task will be used [40]. Participants will be presented with the Swedish words for "high" and "low" with congruent and incongruent high and low tones via wireless headphones (Razer $^{\text {ma }}$ ManO'War), and asked to respond verbally to the corresponding tone as fast as possible. In order to control for cueing effects, stimuli will be presented with a variable interval (1.5-2 s). Reaction times and number of correct answers will be analysed. Participants will walk back and forth on the GAITRite walkway at self-selected speed during six trials of each condition (single- and DT gait). Acceleration and deceleration distances of three meters on each side of the mat will be provided to ensure steady state walking upon the mat itself [41]. The Auditory Stroop task will also be performed in a sitting position as a comparative single task measure. The order of performance of the single versus DT Auditory Stroop task will be randomized. Importantly, participants will be instructed to pay equal attention to both tasks during DT gait, and standardized practice trials of both gait and the Auditory Stroop task during singleand DT conditions will be performed before the assessments. Dual-task interference (DT performance minus single-task performance) will be calculated for both the cognitive and motor interference according to Rochester et al. [42]. Perceived walking ability will be assessed with the Walk-12G questionnaire, which is a 12 item self-reported rating scale for walking difficulties in everyday life [43].

Physical activity will be assessed with a validated physical activity monitor (Actigraph GT3X+, Manufacturing Technology Inc., Fort Walton Beach, FL, USA) and supplemented with a self-rating scale, the Frändin-Grimby activity scale [44]. The physical activity monitor will be used under free-living conditions, worn around the waist for seven consecutive days, as described in earlier work $[45,46]$. Outcomes will be be total physical activity (mean steps per day) and time spent in different intensity levels [47].

Balance confidence during activities of daily living will be assessed with the Swedish version of the Activitiesspecific balance confidence $(A B C)$ scale [48], where confidence to maintain balance during 16 different real-life situations is rated between 0 (no confidence) and 100\% (completely confident).

The Movement Disorders Society Unified PD Rating Scale (MDS-UPDRS) will be used to assess disease related symptoms. The MDS-UPDRS has four parts, I: Non-motor Experiences of Daily Living; II: Motor Experiences of Daily Living; III: Motor Examination; IV: Motor Complications. The scale comprises 65 items altogether [49].

Health related quality of life will be assessed both with a disease specific measurement (the PD Questionnaire, PDQ-39) and with a generic measurement instrument used in a wide range of health conditions and treatments (EQ-5D). EQ-5D consists of five questions/dimensions assessed on a 3 level scale in addition to the evaluation of self-rated health on a vertical visual analogue scale (EQ-VAS) [50]. PDQ-39 is a 39 item questionnaire grouped into eight dimensions of functioning and wellbeing. The patient reports their difficulties as inflicted by PD during the last month on a 5 point scale regarding daily living, including relationships, social situations and communication [51].

Depression and anxiety will be evaluated with the Hospital Anxiety and Depression Scale (HADS); a self-rating scale consisting of two subscales (anxiety and depression) with eight questions relevant to each subscale [52].

To assess training-related cognitive changes, a neuropsychological battery will be performed, assessing a 
range of cognitive domains that are commonly affected in people with PD. Executive functions (mainly inhibition, task-set switching, and initiation) will be assessed with the following: 1) Trail Making Test (TMT) trial 4 from Delis-Kaplan Executive Function System (D-KEFS), 2) the Color-Word Interference Test trial 1-4 from DKEFS, and 3) Verbal Fluency trial 1-3 from D-KEFS [53]. Attention/working-memory (mainly maintenance, manipulation, psychomotor speed, visual search, and sequencing) will be assessed with Digit Span from Wechsler Adult Intelligence Scale - Fourth Edition (WAIS-IV) [54], the TMT trial 1-3, and 5 from D-KEFS. Episodic memory (learning, direct recall, delayed recall, and recognition) will be assessed with Ray Auditory Verbal Learning Test (RAVLT) [55] and Brief Visuospatial Memory Test - Revised (BVMT-R) [56]. Finally, visuospatial functions will be assessed with the Copy condition from BVMT-R.

We will also include speech and voice assessments to evaluate the effects of the control treatment (HiCommunication). The Dysarthria test [57] will be used to assess speech function (including respiration and phonation, oral-motor- and velopharyngeal function and articulation). This test also includes assessment of prosody and intelligibility, as well as a section for self-reported data on perceived speech impairment and communicative participation. In addition, a standardized speech recording, including production of sustained phonation, text reading and production of spontaneous speech will be made in a sound-proofed booth using a head-mounted microphone. Analyses of the speech recording will include voice sound level, mean fundamental frequency and measurements of speech rate, which can also be used for further perceptual analyses.

Magnetic Resonance Imaging (MRI) will be acquired at one site (MRI Center, Karolinska University Hospital, Huddinge), using a 3 Tesla Philips Ingenia and a 15channel coil. Subjects will undergo one MRI session before and one after the training period. Each session will include two structural sequences; a T1 scan (6 min) and a T2 scan (5 min). It will also include two task-evoked fMRI sequences; a visuo-motor task consisting of visually informed finger movements and a DT designed as the visuo-motor task but with the addition of a simple counting task. In the visuo-motor task, four white circles will be shown on a black screen with one circle blinking (turning grey) every $1.2 \mathrm{~s}$. The participants will have a 2button response box in each hand and use their index and middle fingers to respond to the circle turning grey by pressing the corresponding button (see Fig. 2). Both tasks will have a block-design with each block interleaved by a 6-s rest. Each block of the visuo-motor task will consist of 40 visual stimuli (i.e., circles turning grey) and the DT will consist of blocks of 32 visual stimuli. In the DT, the visuo-motor task is combined with a simple counting task in five of the blocks. These five blocks are followed by an extra six seconds where the participant is required to give an answer to the counting task. To make the participant aware of the approaching dual task, these blocks will also be preceded by all four circles shortly turning green.

All participants will be trained outside the scanner on both the visuo-motor and DT with a similar set-up of buttons as used inside the scanner. MR-compatible glasses will be provided when necessary, and earplugs and headphones will always be used to protect against noise. Subjects will be instructed to lie as still as possible and head padding will be used to help subjects keep their heads still.

Brain-derived neurotrophic factor (BDNF) will be assessed from blood serum, before and after the exercise interventions, and analyzed with ELISA commercial analyzing kit. Venous blood will be collected into sampling tubes at a hospital sampling central. The samples will be centrifuged, aliquoted and stored at $-80^{\circ} \mathrm{C}$ until analysis.

To enable comparison between the two groups with regard to motivation, expectancy of symptom relieve and credibility of the intervention, a 10-item questionnaire inspired by Devilly \& Borkovec [58] will be filled in by the participants three weeks into the intervention. After the intervention period, both groups will also answer an anonymous questionnaire regarding the adverse events during the training period, their perception of the therapist-led exercise, and the home exercise.

\section{Ethics and data handling}

The trial has been approved by the Regional Ethical Review Board in Stockholm 2016/1264-31/4, 2017/125832 and 2017/2445-32. Participants will receive written and oral information about the study and all assessments, as well as provide written informed consent before the start of the assessments. Only authorized researchers will have access to the data. Data will be pseudonymized and stored in paper and digital format in accordance with regulations regarding public authority archives and the General Data Protection Regulation.

\section{Sample size}

We calculated the power based on 2000 bootstrap samples from data generated on parameters obtained from pilot data. The pilot data consisted of 12 individuals with PD, 5 from one group, and 7 from the other. By testing the interaction term of a random-intercept model with group (binary), time (binary), and their interaction as covariates, we found that a sample size of 40 individuals with PD per intervention would give a power of $82 \%$ to detect a between-group difference of two points in mean of the total score of the Mini-BESTest at post-testing. 


\section{Task set-up during fMRI}
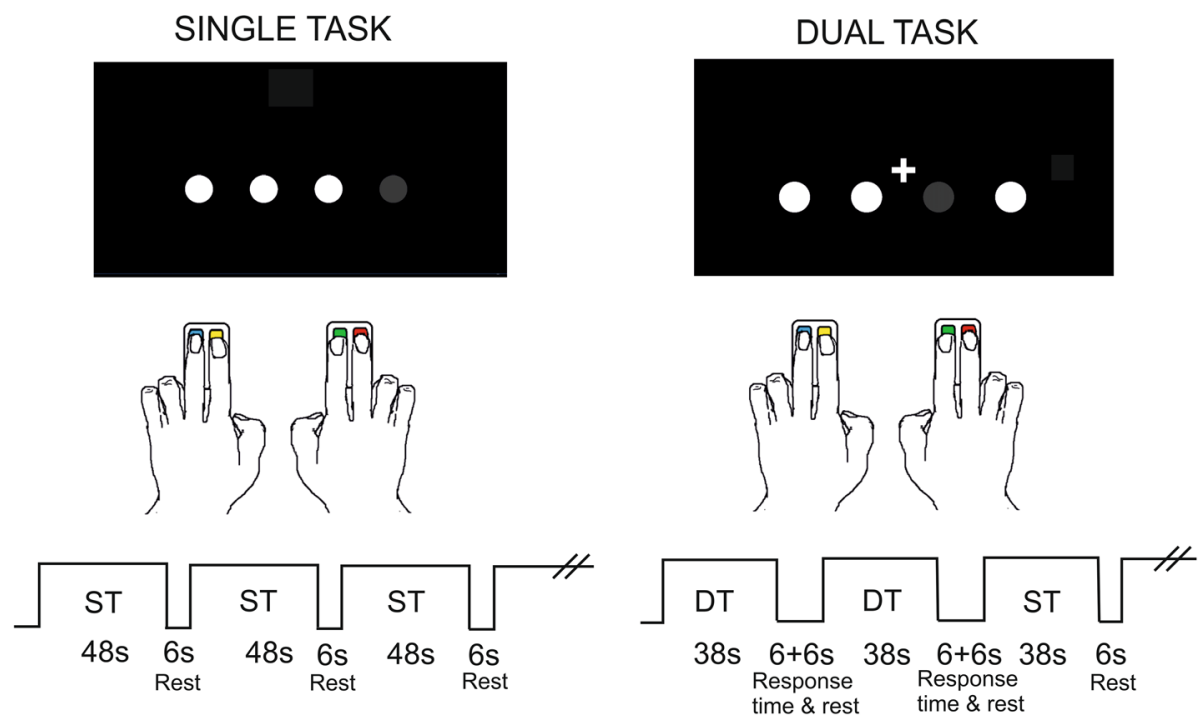

Fig. 2 illustrates the experimental set-up of the single tast (ST) and dual-task (DT) during the functional magnetic resonance imaging (fMRI). During both visu-motor tasks four white circles are shown on a black screen with one circle blinking (turning grey) every $1.2 \mathrm{~s}$. The participants will have a 2-button response box in each hand and use their index and middle fingers to respond to the circle turning grey by pressing the corresponding button. During the DT, participants will also perform a counting task, i.e. count how many white plus sign appearing on the screen during the block. After each DT-block, participants will be given four alternatives and respond with the corresponding button how many plus-signs they have counted

Based upon our previous studies [19, 24], this sample size would also be sufficient to adequately power some of the secondary outcomes (gait velocity, step length and dual-tasking). We also account for expected imaging related exclusions due to technical problems or head/body motions. Taken together, to ensure statistical power, we would need to include 50 in each intervention group.

\section{Statistical analysis}

Descriptive statistics will be used to describe the groups at baseline. We will employ a random effects general linear model analysis to handle unbalanced/missing data. Group (HiBalance and HiCommunication) and time (pre- and post-intervention) will be factors in the model that determine the effects of the interventions. Groupby-time interaction, main effect of group and time analyses will be evaluated. To compare the magnitude of gains, effect size will be calculated.

For the imaging data, we will primarily investigate changes in functional connectivity with a focus on the fronto-striatal network, but with the addition of a whole-brain analysis. To link behavioral changes in balance performance to changes in the brain, we will perform correlational analysis using the Spearman linear correlations within the respective groups. Sub-group analysis in relation to disease stage or baseline cognitive performance might be performed to check for heterogeneity of the effects. In case of skewed distribution of the data, corresponding non-parametric statistics will be used to assess main effects of the intervention. The apriori alpha level for analyses of 0.05 with adjustments for multiple testing will be used when appropriate.

\section{Discussion}

This is the first study to compare the HiBalance program with a double-blinded design and an active control treatment incorporating a similar dose, design and deliverance, which takes into consideration the social aspects of a group treatment and the engagement of health care personnel. Using structural and functional MRI together with levels of BDNF before and after training will allow us to explore neuroplasticity changes due to the exercise interventions from two perspectives. The ultimate goal of this study is to link behavioural effects of a highly challenging balance exercise program to changes in functional connectivity in the fronto-striatal network, as well as blood markers of neurobiological plasticity in people with mild-moderate PD.

The overall trial design has been found to be feasible and acceptable in a pilot study (in manuscript). From the pilot study, we have further strengthened the design by using a double-blinded design, as well as optimized the MRI protocol to avoid diplopia and daytime somnolence, amended the exclusion criteria (e.g. added criteria for blindness, diplopia, tremor, dyskinesia or dystonia), streamlined the blood sampling process and analysis, as 
well as added a participant expectancy and credibility questionnaire.

There are some limitations to the proposed study, although the total dose of ant-Parkinson medication will be monitored from a longitudinal perspective, separating the potential effects of the interventions from those of levodopa medication will be limited. On the other hand, as mentioned in the introduction, the effects of Levodopa on balance, gait and cognition are ambiguous, in that it may worsen gait and balance [13].

This study design will also allow us to explore many other questions regarding motor and cognitive performance and corresponding brain activity, as well as explore the effects of a new speech and communication treatment for people with mild-moderate PD. The knowledge gained from this trial could accelerate the understanding of the link between motor and cognitive function and how motor-cognitive exercise changes the function of the brain, as well as the behaviour. Such results can revolutionize the way we treat people with PD and possibly also other neurodegenerative disorders, as well as provide new hope to patients for a longer life with better health, greater independence and improved quality of life.

\section{Trial status}

Recruitment of participants began in December 2017 and data collection started in January 2018 and is expected to go on until December 2019.

\section{Abbreviations \\ ABC: Activities-specific balance confidence; BDNF: brain-derived neurotrophic factor; BVMT-R: Brief Visuospatial Memory Test - Revised; BVMT-R: Brief Visuospatial Memory Test - Revised; D-KEFS: Delis-Kaplan Executive Function System; DT: dual-task; fMRI: Functional magnetic resonance imaging: HADS: Hospital Anxiety and Depression Scale; LED: Levodopa equivalent daily dose; MDS-UPDRS: Movement disorder society version of the unified Parkinson disease rating scale; Mini-BESTest: Mini Balance Evaluation Systems Test; MoCA: Montreal Cognitive Assessment; MRI: Magnetic resonance imaging; PD: Parkinson's disease; PDQ-39: Parkinson's disease questionnaire; RAVLT: Ray Auditory Verbal Learning Test; RCT: randomized controlled trial; SPIRIT: Standard Protocol Items: Recommendations for Interventional Trials; SRTT: serial reaction time task; TMT: Trail Making Test; WAIS-IV: Wechsler Adult Intelligence Scale - Fourth Edition.}

\section{Acknowledgements}

We would like to acknowledge Professor Matteo Bottai at the Biostatistics Core Facility at Karolinska Institute for assistance with the sample size and statistical analysis section.

\section{Trial status}

The recruitment of participants is still ongoing at the time of manuscript submission.

\section{Authors' contribution}

EF and MBW conceived the idea for this study and designed it along with the other authors. All authors (EF, HJ, MF, UE, MBW, ES, AL, ML, SH, PS and $\mathrm{MH}$ ) were involved in drafting and revising the manuscript. All authors will be involved in data collection, analysis and/or manuscript preparation as the study proceeds. All authors read and approved the final manuscript.

\section{Funding}

This work is peer-reviewed and funded by the Swedish research council (2016-01965). Additional support was provided after peer-review of the proposed study by the Parkinson's Research Foundation, the Regional Agreement on Medical Training and Clinical Research between Stockholm County Council and Karolinska Institutet (ALF) and the Doctoral School in Health Care Science at Karolinska Institutet. The funding sources had no role in the design of this study and will not have any role in the collection, analysis, interpretation of data and writing of scientific manuscripts.

\section{Availability of data and materials}

The datasets generated during and/or analysed during the current study are not publicly available due to Swedish and EU personal data legislation but are available from the corresponding author on reasonable request. Any sharing of data will be regulated via a data transfer and user agreement with the recipient.

\section{Ethics approval and consent to participate}

The trial has been approved by the Regional Ethical Review Board in Stockholm 2016/1264-31/4, 2017/1258-32 and 2017/2445-32. The participants will receive written and oral information about the study and all assessments, as well as provide written informed consent before the start of the assessments.

\section{Consent for publication}

Not applicable

\section{Competing interests}

The authors declare that they have no competing interests.

\section{Author details}

'Department of Neurobiology, Care sciences and Society, Division of Physiotherapy, Karolinska Institutet, Stockholm, Sweden. ${ }^{2}$ Karolinska University Hospital, Allied Health Professionals Function, Function Area Occupational Therapy \& Physiotherapy, Stockholm, Sweden. ${ }^{3}$ Stockholms Sjukhem, R\&D unit, Stockholm, Sweden. ${ }^{4}$ Department of Neurobiology, Care sciences and Society, Division of Clinical Geriatrics, Karolinska Institutet, Stockholm, Sweden. ${ }^{5}$ Karolinska University Hospital, Allied Health Professionals Function, Function Area Medical Psychology, Stockholm, Sweden. ${ }^{6}$ Department of Clinical Science, Intervention and Technology, CLINTEC, Division of Speech and Language Pathology, Karolinska Institutet, Stockholm, Sweden. ${ }^{7}$ Karolinska University Hospital, Allied Health Professionals Function, Function Area Speech and Language Pathology, Stockholm, Sweden. ${ }^{8}$ Department of Neurobiology, Care sciences and Society, Aging Research Center, Karolinska Institutet, Stockholm, Sweden. ${ }^{9}$ Department of Clinical Neuroscience, Division of Neurology, Karolinska Institutet, Stockholm, Sweden. ${ }^{10}$ Department of Health Promoting Science, Sophiahemmet University, Stockholm, Sweden.

Received: 16 August 2019 Accepted: 30 October 2019

Published online: 12 November 2019

\section{References}

1. Schrag A, Jahanshahi M, Quinn N. How does Parkinson's disease affect quality of life? A comparison with quality of life in the general population. Mov Disord. 2000;15(6):1112-8.

2. Jankovic J. Parkinson's disease: clinical features and diagnosis. J Neurol Neurosurg Psychiatry. 2008;79(4):368-76.

3. Lökk J, Borg S, Svensson J, Persson U, Ljunggren G. Drug and treatment costs in Parkinson's disease patients in Sweden. Acta Neurol Scand. 2012; 125(2):142-7.

4. Dirnberger $G$, Jahanshahi M. Executive dysfunction in Parkinson's disease: a review. J Neuropsychol. 2013;7(2):193-224

5. Wu T, Hallett M, Chan P. Motor automaticity in Parkinson's disease. Neurobiol Dis. 2015;82:226-34

6. Ekman U, Eriksson J, Forsgren L, Mo SJ, Riklund K, Nyberg L. Functional brain activity and presynaptic dopamine uptake in patients with Parkinson's disease and mild cognitive impairment: a cross-sectional study. Lancet Neurol. 2012;11(8):679-87. 
7. Cheng FY, Yang YR, Wang CJ, Wu YR, Cheng SJ, Wang HC, Wang RY. Factors influencing turning and its relationship with falls in individuals with Parkinson's disease. PLoS One. 2014;9(4):e93572.

8. Peterson DS, Horak FB. Neural control of walking in people with parkinsonism. Physiology (Bethesda). 2016;31(2):95-107.

9. Kelly VE, Eusterbrock AJ, Shumway-Cook A. A review of dual-task walking deficits in people with Parkinson's disease: motor and cognitive contributions, mechanisms, and clinical implications. Parkinsons Dis. 2012; 2012:918719.

10. Cools R, Barker RA, Sahakian BJ, Robbins TW. Enhanced or impaired cognitive function in Parkinson's disease as a function of dopaminergic medication and task demands. Cereb Cortex. 2001;11(12):1136-43.

11. Franzén E, Paquette C, Gurfinkel VS, Cordo PJ, Nutt JG, Horak FB. Reduced performance in balance, walking and turning tasks is associated with increased neck tone in Parkinson's disease. Exp Neurol. 2009;219(2):430-8.

12. Hall LM, Brauer SG, Horak F, Hodges PW. The effect of Parkinson's disease and levodopa on adaptation of anticipatory postural adjustments. Neuroscience. 2013;250:483-92.

13. Smulders K, Dale ML, Carlson-Kuhta P, Nutt JG, Horak FB. Pharmacological treatment in Parkinson's disease: effects on gait. Parkinsonism Relat Disord. 2016;31:3-13.

14. Tomlinson CL, Patel S, Meek C, Herd CP, Clarke CE, Stowe R, Shah L, Sackley $\mathrm{CM}$, Deane KH, Wheatley K, et al. Physiotherapy versus placebo or no intervention in Parkinson's disease. Cochrane database of systematic reviews. 2013;9:CD002817.

15. Keus S, Munneke M, Graziano M, Paltamaa J, Pelosin E, Domingos J, Bruhlmann S, Ramaswamy B, Prins J, Struiksma C et al: European physiotherapy guidelines for Parkinson's disease. In the Netherlands: KNGF/ ParkinsonNET; 2014.

16. Shen X, Wong-Yu IS, Mak MK. Effects of exercise on falls, balance, and gait ability in Parkinson's disease: a meta-analysis. Neurorehabil Neural Repair. 2016;30(6):512-27.

17. Reynolds GO, Otto MW, Ellis TD, Cronin-Golomb A. The therapeutic potential of exercise to improve mood, cognition, and sleep in Parkinson's disease. Mov Disord. 2016:31(1):23-38.

18. da Silva FC, lop RDR, de Oliveira LC, Boll AM, de Alvarenga JGS, Gutierres Filho PJB, de Melo L, Xavier AJ, da Silva R. Effects of physical exercise programs on cognitive function in Parkinson's disease patients: a systematic review of randomized controlled trials of the last 10 years. PLoS One. 2018; 13(2):e0193113.

19. Löfgren N, Conradsson D, Rennie L, Moe-Nilssen R, Franzén E. The effects of integrated single- and dual-task training on automaticity and attention allocation in Parkinson's disease: a secondary analysis from a randomized trial. Neuropsychology. 2019;33(2):147-56

20. Intzandt B, Beck EN, Silveira CRA. The effects of exercise on cognition and gait in Parkinson's disease: a scoping review. Neurosci Biobehav Rev. 2018; 95:136-69.

21. Petzinger GM, Fisher BE, McEwen S, Beeler JA, Walsh JP, Jakowec MW. Exercise-enhanced neuroplasticity targeting motor and cognitive circuitry in Parkinson's disease. Lancet Neurol. 2013;12(7):716-26.

22. Hirsch MA, van Wegen EEH, Newman MA, Heyn PC. Exercise-induced increase in brain-derived neurotrophic factor in human Parkinson's disease: a systematic review and meta-analysis. Transl Neurodegener. 2018;7:7.

23. Conradsson D, Löfgren N, Ståhle A, Hagströmer M, Franzén E. A novel conceptual framework for balance training in Parkinson's disease-study protocol for a randomised controlled trial. BMC Neurol. 2012:12:111.

24. Conradsson D, Löfgren N, Nero H, Hagströmer M, Ståhle A, Lökk J, Franzén $E$. The effects of highly challenging balance training in elderly with Parkinson's disease: a randomized controlled trial. Neurorehabil Neura Repair. 2015;29(9):827-36.

25. Hoehn MM, Yahr MD. Parkinsonism: onset, progression and mortality. Neurology. 1967;17(5):427-42

26. Nasreddine ZS, Phillips NA, Bedirian V, Charbonneau S, Whitehead V, Collin I, Cummings JL, Chertkow H. The Montreal cognitive assessment, MoCA: a brief screening tool for mild cognitive impairment. J Am Geriatr Soc. 2005: 53(4):695-9

27. Minns Lowe CJ, Wilson MS, Sackley CM, Barker KL. Blind outcome assessment: the development and use of procedures to maintain and describe blinding in a pragmatic physiotherapy rehabilitation trial. Clin Rehabil. 2011;25(3):264-74.
28. Conradsson D, Löfgren N, Ståhle A, Franzén E. Is highly challenging and progressive balance training feasible in older adults with Parkinson's disease? Arch Phys Med Rehabil. 2014;95(5):1000-3.

29. Joseph C, Leavy B, Mattsson S, Falk L, Franzén E. Implementation of the HiBalance training program for Parkinson's disease in clinical settings: a feasibility study. Brain and behavior. 2018;8(8):e01021.

30. Leavy B, Kwak L, Hagströmer M, Franzén E. Evaluation and implementation of highly challenging balance training in clinical practice for people with Parkinson's disease: protocol for the HiBalance effectiveness-implementation trial. BMC Neurol. 2017;17(1):27.

31. Löfgren N, Conradsson D, Rennie L, Moe-Nilssen R, Franzén E. The effects of integrated single- and dual-task training on automaticity and attention allocation in Parkinson's disease: a secondary analysis from a randomized trial. Neuropsychology. 2019;33(2):147-56.

32. Duffy JR. Motor speech disorders: substrates, differential diagnosis and management. 3rd ed. Elsevier Mosby: St Louis, Missouri; 2013.

33. Ho AK, Bradshaw JL, lansek T. Volume perception in parkinsonian speech. Mov Disord. 2000;15(6):1125-31.

34. Tomlinson CL, Stowe R, Patel S, Rick C, Gray R, Clarke CE. Systematic review of levodopa dose equivalency reporting in Parkinson's disease. Mov Disord. 2010:25(15):2649-53.

35. Franchignoni F, Horak F, Godi M, Nardone A, Giordano A. Using psychometric techniques to improve the balance evaluation systems test: the mini-BESTest. J Rehabil Med. 2010;42(4):323-31.

36. King L, Horak F. On the mini-BESTest: scoring and the reporting of total scores. Phys Ther. 2013;93(4):571-5.

37. Löfgren N, Benka Wallen M, Sorjonen K, Conradsson D, Franzén E. Investigating the mini-BESTest's construct validity in elderly with Parkinson's disease. Acta Neurol Scand. 2017;135(6):614-21.

38. Benka Wallen M, Sorjonen K, Löfgren N, Franzén E. Structural validity of the mini-balance evaluation systems test (mini-BESTest) in people with mild to moderate Parkinson disease. Phys Ther. 2016;96(11):1799-806.

39. Löfgren N, Lenholm E, Conradsson D, Ståhle A, Franzén E. The miniBESTest--a clinically reproducible tool for balance evaluations in mild to moderate Parkinson's disease? BMC Neurol. 2014;14:235.

40. Strouwen C, Molenaar EA, Keus SH, Munks L, Bloem BR, Nieuwboer A. Testretest reliability of dual-task outcome measures in people with Parkinson disease. Phys Ther. 2016;96(8):1276-86.

41. Lindemann U, Najafi B, Zijlstra W, Hauer K, Muche R, Becker C, Aminian K. Distance to achieve steady state walking speed in frail elderly persons. Gait Posture. 2008;27(1):91-6.

42. Rochester L, Galna B, Lord S, Burn D. The nature of dual-task interference during gait in incident Parkinson's disease. Neuroscience. 2014;265:83-94.

43. Bladh S, Nilsson MH, Hariz GM, Westergren A, Hobart J, Hagell P. Psychometric performance of a generic walking scale (walk-12G) in multiple sclerosis and Parkinson's disease. J Neurol. 2012;259(4):729-38.

44. Grimby G, Frandin K. On the use of a six-level scale for physical activity. Scand J Med Sci Sports. 2018;28(3):819-25.

45. Nero H, Benka Wallen M, Franzén E, Ståhle A, Hagströmer M. Accelerometer cut points for physical activity assessment of older adults with Parkinson's disease. PLoS One. 2015;10(9):e0135899.

46. Wallen MB, Franzén E, Nero H, Hagströmer M. Levels and patterns of physical activity and sedentary behavior in elderly people with mild to moderate Parkinson disease. Phys Ther. 2015;95(8):1135-41.

47. Hagströmer M, Franzén E. The importance of physical activity and health for physical therapy. Phys Ther Rev. 2017;22(3-4):116-23.

48. Powell LE, Myers AM. The activities-specific balance confidence (ABC) scale. J Gerontol A Biol Sci Med Sci. 1995;50A(1):M28-34.

49. Goetz CG, Tilley BC, Shaftman SR, Stebbins GT, Fahn S, Martinez-Martin P. Poewe W, Sampaio C, Stern MB, Dodel R, et al. Movement Disorder Societysponsored revision of the unified Parkinson's disease rating scale (MDSUPDRS): scale presentation and clinimetric testing results. Mov Disord. 2008; 23(15):2129-70.

50. Burstrom K, Sun S, Gerdtham UG, Henriksson M, Johannesson M, Levin LA Zethraeus N. Swedish experience-based value sets for EQ-5D health states. Qual Life Res. 2014;23(2):431-42.

51. Fitzpatrick R, Peto V, Jenkinson C, Greenhall R, Hyman N. Health-related quality of life in Parkinson's disease: a study of outpatient clinic attenders. Mov Disord. 1997;12(6):916-22.

52. Rodriquez-Blazquez C, Frades-Payo B, Forjaz MJ, de Pedro-Cuesta J, Martinez-Martin P. Longitudinal Parkinson's disease patient study G: 
psychometric attributes of the hospital anxiety and depression scale in Parkinson's disease. Mov Disord. 2009;24(4):519-25.

53. Delis D, Kaplan E, Kramer J. The Delis-Kaplan executive function system: examiner's manual: the psychological corporation; 2001.

54. Weschler D: Wechsler Adult Intelligence Scale - Fourth Edition: Stat Solut. : 2008.

55. Schmidt M. Rey auditory verbal learning test: a handbook: Western psychological services; 1996.

56. Benedict R, Schretlen D, Groninger L, Dobraski M, Shpritz B. Revision of the brief Visuospatial memory test; 1996.

57. Hartelius L. Dysartritest. Studentlitteratur AB: Lund; 2015.

58. Devilly GJ, Borkovec TD. Psychometric properties of the credibility/ expectancy questionnaire. J Behav Ther Exp Psychiatry. 2000;31 (2):73-86,

\section{Publisher's Note}

Springer Nature remains neutral with regard to jurisdictional claims in published maps and institutional affiliations.

Ready to submit your research? Choose BMC and benefit from:

- fast, convenient online submission

- thorough peer review by experienced researchers in your field

- rapid publication on acceptance

- support for research data, including large and complex data types

- gold Open Access which fosters wider collaboration and increased citations

- maximum visibility for your research: over $100 \mathrm{M}$ website views per year

At BMC, research is always in progress.

Learn more biomedcentral.com/submissions 